\title{
People with multimorbidity in outpatient care: patient-focused and needs-oriented healthcare management (MamBo) - protocol for a multiperspective evaluation study
}

Simone Richter ${ }^{1 *}$, Ibrahim Demirer ${ }^{1}$, Kyung-Eun Choi ${ }^{1}$, Johannes Hartrampf ${ }^{2}$, Holger Pfaff $^{1}$ and Ute Karbach ${ }^{3}$

\begin{abstract}
Background: With demographic change, the number of noncommunicable diseases, chronic diseases and multimorbidity is increasing, and so is the demand for health services. This represents a further challenge for the healthcare system. An adequate and efficient treatment of multimorbid patients requires a well-structured, informed and cross-indicated treatment. Therefore, a new form of coordinated, managed and cross-sectoral care for multimorbid patients - the "MamBo" care model - has been developed. Along with the implementation of MamBo, a process and outcome evaluation will be carried out, which is described in this study protocol. The aim of the study is to evaluate the care model according to its implementation process and effectiveness.

Methods: The MamBo-care model will be evaluated in multi-perspective terms. Thus, a process and outcome evaluation with several data sources will be conducted: (1) Annual focus groups and individual interviews with those involved in the process. (2) Various primary data, including surveys of patients, physicians and practice staff at the time of enrolment and 1 year later to enable pre-post comparison. (3) Claim data from the health insurance of the MamBo population in comparison to a comparative population, formed by the propensity score matching method. (4) Process data of the care management. The analysis of qualitative data will be carried out by content analysis according to Mayring. For the analysis of the quantitative data, multivariate analyses are planned.

Discussion: A new form of coordinated care has been introduced to improve intersectoral care of multimorbid patients and reduce the workload on physicians. The effects of the MamBo care model are being investigated for patients, physicians and the cost units. The results will form the basis for the decision whether the care model should be transferred to standard care and what needs to be taken into account for implementation.
\end{abstract}

Trial registration: The study was retrospectively registered in the German Register for Clinical Studies (DRKS00014047) on June 28, 2019.

Keywords: Multimorbid patients, Primary care, Outpatient care, Care management, House calls

\footnotetext{
* Correspondence: Simone.Richter@uk-koeln.de

${ }^{1}$ Institute of Medical Sociology, Health Services Research, and Rehabilitation

Science (IMVR), Faculty of Human Sciences and Faculty of Medicine,

University of Cologne, Eupener Strasse 129, 50933 Cologne, Germany

Full list of author information is available at the end of the article
} 


\section{Background}

In the context of demographic change, one of the most important tasks in the future will probably be to guarantee an adequate care for chronically ill and multimorbid patients [1]. As a growing life expectancy is accompanied by increasing life years and multimorbidity, the ageing society is likely to pose further challenges to the health care system [2]. In Germany, the number of people aged 65 and over is rising constantly while every second person at the age of 65 suffers at least from one chronic illness [3]. Multimorbidity is associated with an increased contact with doctors, more frequent and longer hospital stays and an increased number of drug prescriptions (polypharmacy) [4, 5].

The health care of chronically ill and multimorbid patients is very complex, as several physicians and further health as well as social professionals need to be involved in their care. The integration of general medical and specialist care, outpatient, inpatient and nursing care poses a challenge for the health care but in particular for the general practitioners (GP). They are entrusted with the coordination and, thus, they must always be well informed and communicate with all the professionals involved [1]. Adequate and efficient treatment, therefore, requires well -structured, -coordinated and -informed care. However, the highly complex health care system in Germany makes coordinated action more difficult due to its strong segmentation into the outpatient, hospital and nursing care sectors. In addition, there is no overall legal regulation in Germany for gatekeeper strategies. The given structural conditions of primary and secondary care are accompanied by interface problems between the care sectors and between occupational groups in the outpatient sector [6]. The advantages of structured care are proven by the experiences with the Disease Management Programmes (DMP) in Germany [7]. However, DMPs only address isolated disease. There are currently no structures that address the needs of multimorbid patients.

With the Innovation Fund of the Federal Joint Committee of Germany, new models of care that go beyond the regular care are promoted. Every funded study is evaluated under everyday conditions [8]. The findings of the evaluation serve the Federal Joint Committee and the national legislator as a basis for decision-making. The aim is to transfer successful care models to standard health care.

Building upon this, a new form of coordinated, crosssectoral care for multimorbid patients was developed which is currently being implemented and evaluated. The project supported by the G-BA Innovation Fund is titled: "People with Multimorbidity in Outpatient Care: Patient-Focused and Needs-Oriented Healthcare Management (MamBo)". MamBo consists of a care management
(CM), including responsible persons for the management and up to five monitoring and coordination assistants (MoniKa) set up in a collaborating Regional Health Network (RGL) and a demand management (DM) established on the part of collaborating health insurance. The MoniKas are working on a cross-practice basis and taking over patient-oriented and coordinative tasks (e.g. house calls, patient training, coordination tasks) that can be delegated by doctors. In a continuous improvement process (CIP), the CM and DM, together with the medical practices involved, develop solutions for collective and patients oriented needs (see Fig. 1). An external organisational consulting company supports the initiators of the care model in the implementation.

The overarching objective of this study is to evaluate the care model MamBo in multi-perspective terms according to its implementation process and effectiveness. This raises the question of which factors seem to be beneficial or inhibiting for the implementation of the innovation and whether the quality and efficiency of care in the region have changed over time. The results will be used in political decision-making to assess whether the model for its transfer to general health care is sufficient, appropriate, economic and necessary.

\section{Methods/design \\ Setting}

The new care model MamBo is introduced in a physician's network in the region of Leverkusen, a small metropolis in North Rhine-Westphalia (Germany). The implementation takes place in established general practitioners or specialist practices. Only physicians who are part of the physicians' network can participate in MamBo. Currently, it is estimated that approx. 40 physicians and approx. 160 practice staff will participate in the care model. Inturn, approx. 2615 multimorbid patients with at least three chronic diseases and insured with PronovaBKK will be recruited by the participating physicians for the MamBo program. Patients in oncological or palliative treatment are excluded. The initial period of the MamBoproject was set for 3 years (07.2017-06.2020). After applying for an extension of 9 months, due to delays at the start of the study and initial recruitment difficulties, the study now ends on 31 March 2021.

The presented study is a multiperspective evaluation study including a qualitative and a quantitative evaluation design. Qualitative data for process evaluation and quantitative data for outcome evaluation are collected simultaneously. Different domains of the quantitative and qualitative evaluation design are listed in Table 1. 


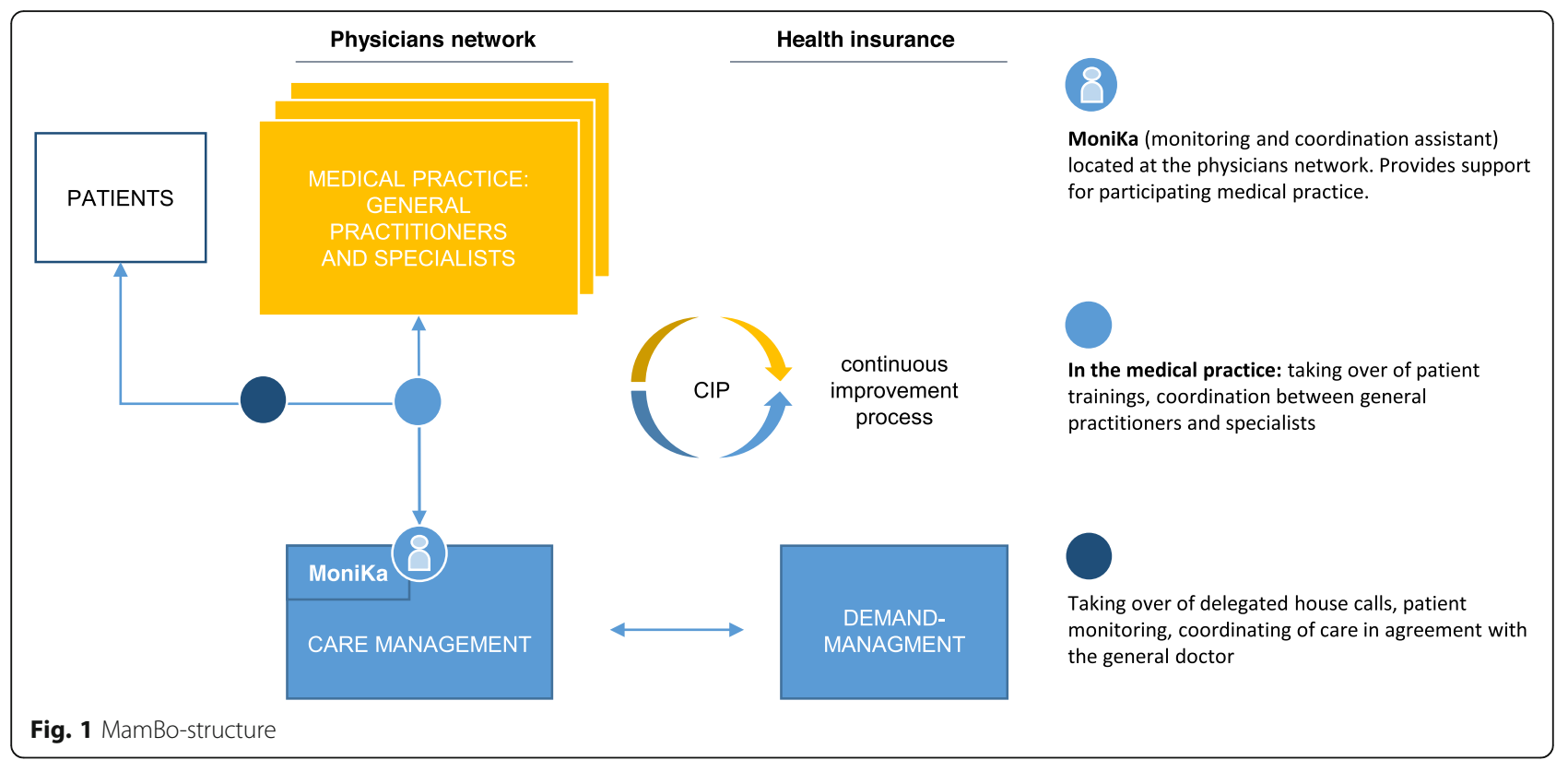

\section{Qualitative evaluation \\ Qualitative evaluation design}

Annual focus groups with participating physicians will be conducted to identify factors influencing the implementation and adoption of the innovation. Additionally, the operative actors will be interviewed. In this context focus groups with all employed MoniKas are held each year to gain deep insights into the role of the MoniKas and the complexity of the delegation process. Along with them, annual face-to-face interviews with the DM and the CM will be conducted to discuss the challenges associated with the implementation on a conceptual and organisational level. In addition, one-time expert interviews with representatives of the management consultancy provide deep insights into the complexity, challenges and benefits of change management as an implementation strategy in health care.

\section{Sampling}

The recruitment of all respondents is conducted purposely [9]. The selection of physicians for the focus groups is based on the Theory Rogers "Diffusion of innovation" [10]. Focus groups with physicians that are active from the beginning ("early adopters", within the first 6 months) are conducted separately from physicians that become active late ("late adopters"). The board of the RGL provides access to physicians relevant for the respective focus groups. Comprehensively, the research

Table 1 Different domains of the multiperspective evaluation study

\begin{tabular}{|c|c|c|c|}
\hline Evaluation type & Data source & & time of data collection \\
\hline \multirow[t]{5}{*}{ Process evaluation } & \multirow[t]{2}{*}{ focus groups } & participating physicians & \multirow[t]{4}{*}{ annual, during the intervention phase } \\
\hline & & monitoring and coordination assistants & \\
\hline & \multirow{3}{*}{$\begin{array}{l}\text { semi- structured } \\
\text { interviews }\end{array}$} & demand management & \\
\hline & & care management & \\
\hline & & management consultancy & once after the implementation \\
\hline \multirow[t]{6}{*}{ Outcome evaluation } & \multirow[t]{4}{*}{ primary data } & questionnaire of participating physicians ( $n \sim 40$ ) & \multirow[t]{3}{*}{ two time-points during the intervention phase } \\
\hline & & $\begin{array}{l}\text { questionnaire of participating practice staff } \\
(n \sim 160)\end{array}$ & \\
\hline & & $\begin{array}{l}\text { questionnaire of participating patients (t0: } n \sim 2460 \text {; } \\
\text { t0 \& t1: } n \sim 1500 \text { ) }\end{array}$ & \\
\hline & & $\begin{array}{l}\text { questionnaire of non-participating physicians } \\
\text { within the physician's network }(n \sim 60)\end{array}$ & one time-point during the intervention phase \\
\hline & \multirow[t]{2}{*}{ secondary data } & $\begin{array}{l}\text { statutory health insurance claim data (group 1: } \\
n \sim 1.975 \text {; group } 2: n \sim 2524 \text { ) }\end{array}$ & \multirow[t]{2}{*}{ during and at the end of the intervention phase } \\
\hline & & RGL- process data & \\
\hline
\end{tabular}


team sends out invitations, information about the procedure and the letters of consent to all participants of face-toface interviews and focus groups via fax or e-mail.

\section{Data collection and analysis}

The focus groups and interviews will follow a semistructured guideline to allow comparisons. For each main topic, open questions are designed. The main topics followed are based on questions regarding inhibiting \& facilitating factors of the implementation, expected and perceived advantages, communication and cooperations, work organisation as well as the usefulness of change management for implementation. The guideline will be adopted according to the background and function of the interviewee.

According to process evaluation standards, the interviews are carried out in waves at different times of the projects' progress. Thus, three focus groups (4-8 physicians each) with participating physicians are planned. One was carried out in June 2018 already and one in January 2019. The second focus group has been supplemented by three individual interviews with doctors in order to gain a deeper insight into the complexity of the implementation and to reach more doctors to share their opinions. If less than four physicians can be recruited for a joint appointment, but other physicians have expressed their interests, additional individual interviews will also be conducted in further data collection waves. The first focus group with MoniKas was carried out in summer 2019 , as only at this time an appropriate number of MoniKas with sufficient MamBo-experience $(n=3)$ was available. After a delay in setting up the MamBostructures, the first interviews with the DM and CM were conducted from May until July 2019. Two management consultants were invited to an interview once in summer 2019.

All interviews will be recorded and transcribed verbatim and pseudonymised [11]. The data will be analysed using qualitative content analysis according to Mayring $[12,13]$. For this, the software MAXQDA will be used.

\section{Quantitative evaluation \\ Quantitative evaluation design}

In order to measure effectiveness and treatment effects, postal survey data of all physicians, practice staff as well as patients enrolled in MamBo, will be conducted as a longitudinal study with two time-points. The first questionnaire (t0) is surveyed immediately after the enrolment into the project. The second questionnaire ( $t 1)$ was originally surveyed 1 year later. As we had difficulties in reaching the required number of patients, the funder proposed to change the intervention period from 1 year to 6 months in the course of the extension application. Due to the fact that the majority of the MoniKa intervention takes place within the first 6 months after enrolment in MamBo and that the MamBo structure is more established in 2020 than in previous years, the evaluation team assumes that a comparison of the groups is still possible and agreed to shorten the period of patient examination. Patients enrolled after November 2019 will, therefore, receive the $\mathrm{t} 1$ after 6 months.

Depending on the doctors' assessment of the patients' health condition and needs as well as the patients' wishes, the included patients receive either a MoniKahome visit, a short MoniKa-call or no MoniKa contact at all. The latter do not receive an additional new form of care and will be considered as the non-treatment group. However, it is expected that they profit indirectly from the MamBo structures on the organisational level. Thus, the patients' survey can be defined as a cohort study with follow-up and a non-randomized treatment. Non-participating physicians will also be surveyed crosssectional.

To evaluate the cost-efficiency and effectiveness of the care model secondary claim data will be used. For this, a quasi-experimental cohort study will be conducted. Multimorbid patients (control group) will be compared with multimorbid MamBo-patients (intervention group).

In addition, documentations of the physician network with records of the process-data of the CM provides information about the implementation process.

\section{Questionnaire data \\ Sampling}

Participants will be approached through the data trustee of the evaluation team, who is the only one with access to the physicians' practice and patients' contact addresses for the surveys. With their enrolment into the MamBo-care model, each participating physician, practice staff and patient receive a written letter of consent to participate in the first and second surveys. Addresses of physicians who are members of the physicians' network but do not participate in MamBo will be provided by the $\mathrm{CM}$ to the trustee. The written declaration of consent and the questionnaire will be sent out in parallel. In order to guarantee high quality, standards for the questionnaire development [14-16] methods of pretesting [17] and the Dillman's Total Design Method for achieving a possibly high response rate [18] were used.

Since the given consent of the physicians' survey in the first study year was approx. $50 \%$ and is a lot lower in the practice staff survey, a response from approx. 20 and 40 participants are expected for t0. The first questionnaire of all participating physicians and their practice staff started in the first half of 2018. The nonparticipating physicians survey was conducted in summer $2019(n \sim 60)$. 
The t0 questionnaire of patients shows a very high consent rate and a response rate of approx. 80\%. With the high response rate and incentives provided, low panel attrition is expected (approx. 20\%). As an incentive, a postage stamp is enclosed with the t1 questionnaire. The survey of patients also started in the first half of 2018. The last enrolment for the t0 questionnaire will be in April 2020 ( $\mathrm{n} \sim 1900$ ), accordingly approx. 1500 patients will be surveyed at both, $\mathrm{t} 0$ and $\mathrm{t} 1$.

\section{Data collection and analysis}

To determine the perceived benefits of the care model the physician survey includes expectations of the care model ( $\mathrm{t} 0)$ or rather the fulfilment of expectations (t1). Besides that, practice characteristics (e.g. number of MFAs, joint practice/single practice) and process data (e.g. workload, information procurement) are collected. In the survey of non-participating physicians, reasons for non-participation are collected additionally. By distinguishing between participating and non-participating physicians, but also between "early adopters" and "late adopters", it can be determined whether and if so which characteristics are significantly different between these groups. With the second survey, a pre-post comparison is possible to examine which factors have changed as a result of participation.

With the first patient survey (t0), sociodemographic characteristics, health-related characteristics (e.g. state of health, mobility, well-being), general characteristics (e.g. social support, coping, living environment), satisfaction with previous care, reasons for participating in MamBo and expectations are recorded. Translated and validated standard scales used in the physicians and patients surveys are listed in Table 2.

In the second survey (t1) only the time-variant measures remain. In addition, questions regarding life events and the MamBo-intervention are added. Primary outcomes are patients' perceptions of the quality of care, social support, drug compliance and wellbeing. The analysis of the survey at t0 examines whether and in which characteristics participating patients differ from each other at their point of enrolment. With the second survey, a pre-post comparison within one group and a pre-post comparison between the treatment groups and the non-treatment group will be possible.

For data preparation, Tele-form ${ }^{\circ}$ software has been used. The quantitative data will be analysed using regression-based methods. Structural Equation Modeling (SEM) is used to study intermediate social factors. Difference-In-Differences (DiD) estimation are used to inspect the effect of the MoniKa intervention on e.g. patient satisfaction. Confounder adjustment will be achieved by using inverse-probability weights (IPW), to adjust for the probability of being visited by a MoniKa (treatment group). Various covariates will be specified as confounders like patients' morbidity characteristics (patients' mobility, age and years of being chronically ill). In addition, the patients' trust in the physicians will also be accounted for. To assess comparability, a subgroup analysis will be performed between patients who completed the $t 1$ questionnaire after 1 year and patients who received it after 6 months. Data will be analysed using StataMP 15.1.

Table 2 Standardized scales used in the postal surveys

\begin{tabular}{ll}
\hline Scale & Outcome \\
\hline Physicians survey & \\
HSOPS_M Hospital Survey on Patient Safety Culture for Hospital Management - & patient safety \\
overall perceptions of safety) [19] & \\
Dispositional Resistance to Change Scale - routine seeking [20] & openness to innovations \\
The workload in Nursing scale captures - psychophysical overload [21] & psychophysical burden of workload \\
Patients survey & \\
WHO 5-items Well-being Scale [22] & wellbeing \\
PACIC - Patient Assessment of Chronic Illness Care shortened form [23] & $\begin{array}{l}\text { patients' perceptions of the quality of care they have } \\
\text { received for their chronic conditions }\end{array}$ \\
$\begin{array}{l}\text { EFK-HPC Questionnaire on Disease Processing - Acting, problem-oriented } \\
\text { Coping [24] }\end{array}$ & current coping efforts \\
Patient questionnaire of cologne (subscale) [25] & burden of disease \\
HL-COM -Health Literacy sensitive Communication [26] & health literacy sensitive communication \\
Medication Adherence Rating Scale (MARS) [27] & drug compliance \\
BS6 - Brief Social Support Scale (BS6) [28] & social support \\
PHQ-2-Patient Health Questionnaire 2 [29] & depression \\
EORTC-QLQ-C30 (subscales) [30] & quality of life regarding physical function and global health \\
\hline
\end{tabular}




\section{SHI-claim data \\ Sampling}

Claim data will be used to assess and compare the costs and use of health services between MamBo patients and patients in standard care. For this purpose, the SHI "PronovaBKK" provides anonymised claim data of the MamBopopulation. A statistical twin with data available to the German Health Risk Institute (HRI) [31] is determined for each MamBo participant using the propensity score matching (PSM) method [32]. Matching is performed by the HRI according to criteria such as age, gender and diagnosis.

A sample size of $n=2617$ corresponding to a statistical power of $79.2 \%$ was aimed, given an unpaired t-test, a Cohen's d of 0.068 and a significance level of 0.05 . However, the patients' enrolment did not correspond to the expected sample size goals, which caused reduced statistical power. To ensure maximum statistical power, the evaluation design of the SHI-claim data analysis has been adopted to the different temporal availabilities of the key parameters. One part can be delivered within 3 months. The other part is available with a delay of up to 9 months. Therefore, group $1(n=1.975)$ was closed on 01.04.2019 and group $2(n=2365)$ was closed on 01.10.2019. By this, sufficiently high power is guaranteed. The power for Group 1 is about $68 \%$ and for Group 2 about $76 \%$.

\section{Data collection and analysis}

Both patient-related (e.g. improvements of care,) as well as payer-related (e.g. hospitalizations, utilization of outpatient care) objectives will be considered in the analysis. With MamBo a mean cost reduction of $12,5 \%$ is expected due to reduced hospitalization rates. For all variables, a comparison is made between the intervention group and the twin group with significance tests. The selection of the appropriate statistical test depends, among others, on the type (binary/continuous) and distribution of the respective variables.

\section{RGL process data (physician's network)}

The number of participating physicians and of patients they enroll, all processes of the CM including activities of the MoniKa and the number and contents of the continuous improvement process meetings are documented in the RGL. This data is handed in quarterly to the evaluating institute in aggregated form. This data enables the evaluation of the success of the implementation determined by successful MoniKa delegation, quality and quantity of MoniKa visitations and sustainable physicians' participation.

\section{Discussion}

The "MamBo" care model offers a new form of coordinated, managed and cross-sectoral care for multimorbid patients. There are already coordinated programmes such as the DMPs in Germany [7]. While DMPs only promote isolated diseases, MamBo addresses the needs of multimorbid patients. This study evaluates a new form of care formatively and summatively. By conducting a multiperspective study with several data sources and study results, patient-, provider- and cost-unit-related goals are addressed. In addition, confounding factors are taken into account by considering various additional data sources, such as process data and SHI claim data. As far as we know, the study will also be the first to formatively evaluate the benefits of a management consultancy that supports the change management as an implementation strategy for complex health innovations.

However, it should be noted that this study is a health services research study. Thus, this is an evaluation study that primarily investigates the effectiveness of a new care model. Consequently, the study shows its limitations. There will be an increased risk of selection bias due to the quasiexperimental study design in which participating physicians enroll patients and only selected patients receive MoniKa treatment. Additionally, within the formative evaluation just willing and thus mostly active physicians can be interviewed. Due to difficulties in the recruitment process, the evaluation-design had to be adopted during the project period. The occurrence of group differences between patients who completed the $t 1$ survey after one year and patients who were interviewed after a six-month intervention cannot be completely excluded.

\section{Abbreviations \\ CM: Care Management; CIP: Continuous Improvement Process; DM: Demand Management; DiD: Difference-In-Differences; DMP: Disease Management Programme; GP: General Practitionairs; HRI: German Health Risk Institute; IPW: Inverse-Probability Weights; MoniKa: Monitoring and Coordinating Assistant; MamBo: People with Multimorbidity in Outpatient Care: Patient- Focused and Needs-Oriented Healthcare Management"; PSM: Propensity Score Matching; RGL: Regional Health Network; SHI: Statutory Health Insurance; SEM: Structural Equation Modeling}

\section{Acknowledgments}

We would like to thank our student assistants at the Institute of Medical Sociology, Health Services Research and Rehabilitation Science (IMVR) for their assistance in designing the study protocol. We gratefully acknowledge the support and cooperation within the MamBo-project.

\section{Authors' contributions}

HP, UK and JH designed the study. The scientific management is governed by HP and UK. They are responsible for the study design and project management. The responsibility of the operative management is delegated to ID. While ID is the principal investigator of the summative evaluation, SR is the principal investigator of the formative evaluation. SR drafted the manuscript with support of ID, UK and K-EC. The final manuscript has been read and approved by all authors.

\section{Funding}

This study is externally funded by the Innovation Fund of the Federal Joint Committee, the G-BA, grant number 01NVF17001. The funding institution does not have an active role in the study. The study has passed a peerreview selection process.

\section{Availability of data and materials}

It is planned to submit the results of the formative and summative evaluation for publication in peer-reviewed journals and to present them at national and international conferences. The dissemination will also be supported by professional 
public relations activities. The anonymous datasets generated during the current study may be made available from the corresponding author on a reasonable request. Protocol modifications will be communicated to relevant parties.

\section{Ethics approval and consent to participate}

On behalf of all the trial sites, the study has been approved by the Ethics Committee of both the Medical Faculty University Hospital of Cologne as well as the North Rhine Medical Association and is registered at the German clinical trials register (DRKS00014047). Before participating in the study, all participants give written consent to conduct the first and second postal survey and, in case of an invitation, to participate in the interviews or focus groups. Participants of the interviews and focus groups will be informed in advance about the procedure and the guarantee of data protection in compliance with the regulations. All participants will be informed about the possibility to drop out of the study at any time, without negative consequences and to deny answering questions. The first surveys will be pseudonymised, the second surveys will be conducted anonymously. The numerical pseudonymisation and personal identifiers will be recorded by the data protection officer of the IMVR and will only be recorded to manage the responses to the questionnaire. The operative researchers do not have access to this data. Thus, a complete anonymous data import and data analysis can be guaranteed. All information will be kept confidential, address data will not be passed on to third parties. The pre-test after the think-aloud method is carried out with every postal survey before dispatch. Claims data will be transmitted anonymously by PronovaBKK. Process data will be transmitted anonymously by the RGL.

\section{Consent for publication}

The manuscript does not contain any individual person's data in any form.

\section{Competing interests}

Prof. Dr. Holger Pfaff is an Associate Editor in BMC Health Services Research. Furthermore, the authors declare no conflicts of interest.

\section{Author details}

'Institute of Medical Sociology, Health Services Research, and Rehabilitation Science (IMVR), Faculty of Human Sciences and Faculty of Medicine, University of Cologne, Eupener Strasse 129, 50933 Cologne, Germany. ${ }^{2}$ Professional association of ophthalmologists in Germany e.V, Düsseldorf, Germany. ${ }^{3}$ Faculty of Rehabilitation Sciences, TU Dortmund University, Dortmund, Germany.

Received: 26 February 2020 Accepted: 31 March 2020

Published online: 13 April 2020

\section{References}

1. Sachverständigenrat zur Begutachtung der Entwicklung im Gesundheitswesen. Koordination und Integration - Gesundheitsversorgung in einer Gesellschaft des längeren Lebens: Sondergutachten 2009 Kurzfassung. Baden-Baden: Nomos-Verl-Ges; 2009.

2. Tetzlaff J, Muschik D, Epping J, et al. Expansion or compression of multimorbidity? 10-year development of life years spent in multimorbidity based on health insurance claims data of Lower Saxony, Germany. Int J Public Health. 2017;62(6):679-86. https://doi.org/10.1007/s00038-017-0962-9.

3. Robert Koch Institut. Demografische Alterung und Folgen für das Gesundheitswesen. 3rd ed. Berlin: GBE Kompakt; 2012.

4. Junius-Walker $\mathrm{U}$, Theile G, Hummers-Pradier E. Prevalence and predictors of polypharmacy among older primary care patients in Germany. Fam Pract. 2007;24(1):14-9. https://doi.org/10.1093/fampra/cml067.

5. Sundmacher L, Schüttig W, Faisst C Krankenhausaufenthalte infolge ambulant-sensitiver Diagnosen in Deutschland http://www.zi.Ode/cms/ fileadmin/images/content/PDFs_alle/3-. Accessed 17 June 2019.

6. Simon M. Das Gesundheitssystem in Deutschland: Eine Einführung in Struktur und Funktionsweise. 6th ed. Bern: Hogrefe; 2017.

7. Bundesversicherungsamt. Bericht des Bundesversicherungsamtes zur vergleichenden Evaluation von strukturierten Behandlungsprogrammen bei Diabetes mellitus Typ 2. Bonn; 2009.

8. Pfaff $\mathrm{H}$, Neugebauer $\mathrm{E}$, Glaeske $\mathrm{G}$, et al. Lehrbuch Versorgungsforschung: Systematik - Methodik - Anwendung; mit 19 Tabellen. Stuttgart: Schattauer; 2011.

9. Patton MQ. Qualitative research \& evaluation methods. 3rd ed. Thousand oaks: Sage; 2009.
10. Rogers EM. Diffusion of innovations, Free Press trade paperback edition. Social science. 5th ed. New York, London, Toronto, Sydney: Free Press; 2003.

11. Fuß S, Karbach U. Grundlagen der Transkription: Eine praktische Einführung. utb-studi-e-book, vol. 4185. Opladen, Stuttgart: Budrich; UTB; 2014.

12. Mayring P (2008) Qualitative Inhaltsanalyse: Grundlagen und Techniken, 10th ed., Beltz Pädagogik. Beltz.

13. Gläser J, Laudel G. Experteninterviews und qualitative Inhaltsanalyse als Instrumente rekonstruierender Untersuchungen. 4. Auflage ed. Wiesbaden: Lehrbuch. VS Verlag; 2010.

14. Schaeffer NC, Presser S. The science of asking questions. Annu Rev Sociol. 2003;29(1):65-88. https://doi.org/10.1146/annurev.soc.29.110702.110112.

15. Schaeffer NC, Dykema J. Questions for surveys: current trends and future directions. Public Opin Q. 2011;75(5):909-61. https://doi.org/10.1093/poq/nfr048,

16. Bradburn NM, Sudman S, Wansink B. Asking questions: the definitive guide to questionnaire design - for market research, political polls, and social and health questionnaires. Research methods for the social sciences. Hoboken: Wiley; 2004.

17. Prüfer $P$, Rexroth $M$. Z Zwei Phasen-Pretesting. In: Mohler PP, editor. Querschnitt: Festschrift für Max Kaase. Mannheim: ZUMA; 2000. p. 203-19.

18. Dillman DA. Mail and telephone surveys: the total design method. A Wileyinterscience publication. New York: Wiley; 1978.

19. Hammer A, Ernstmann N, Ommen O, et al. Psychometric properties of the hospital survey on patient safety culture for hospital management (HSOPS_M). BMC Health Serv Res. 2011;11:165. https:/doi.org/10.1186/1472-6963-11-165.

20. Oreg $\mathrm{S}$, Bayazit M, Vakola $\mathrm{M}$, et al. Dispositional resistance to change: measurement equivalence and the link to personal values across 17 nations. J Appl Psychol. 2008;93(4):935-44. https://doi.org/10.1037/0021-9010.93.4.935.

21. Bartholomeyczik E Arbeitsbelastung in der Krankenpflege. ZIS - GESIS Leibniz Institute for the Social Sciences.

22. Topp CW, Østergaard SD, Søndergaard S, et al. The WHO-5 well-being index: a systematic review of the literature. Psychother Psychosom. 2015; 84(3):167-76. https://doi.org/10.1159/000376585.

23. Goetz K, Freund T, Gensichen J, et al. Adaptation and psychometric properties of the PACIC short form. Am J Manag Care. 2012;18(2):e55-60.

24. Franke GH, Mähner N, Reimer J, et al. Erste Überprüfung des Essener Fragebogens zur Krankheitsverarbeitung (EFK) an sehbeeinträchtigten Patienten. Zeitschrift für Differentielle und Diagnostische Psychologie. 2000; 21(2):166-72. https://doi.org/10.1024//0170-1789.21.2.166.

25. Pfaff $\mathrm{H}$, editor. Der Kölner Patientenfragebogen (KPF): Entwicklung und Validierung eines Fragebogens zur Erfassung der Einbindung des Patienten als Kotherapeuten. Sankt Augustin: Asgard-Verl.; 2003.

26. Ernstmann N, Halbach S, Kowalski C, et al. Measuring attributes of health literate health care organizations from the patients' perspective: development and validation of a questionnaire to assess health literacysensitive communication (HL-COM). Z Evid Fortbild Qual Gesundhwes. 2017; 121:58-63. https://doi.org/10.1016/j.zefa.2016.12.008.

27. Mahler $\mathrm{C}$, Hermann $\mathrm{K}$, Horne $\mathrm{R}$, et al. Assessing reported adherence to pharmacological treatment recommendations. Translation and evaluation of the medication adherence report scale (MARS) in Germany. J Eval Clin Pract. 2010;16(3):574-9. https://doi.org/10.1111/j.1365-2753.2009.01169.x.

28. Beutel ME, Brähler E, Wiltink J, et al. Emotional and tangible social support in a German population-based sample: development and validation of the brief social support scale (BS6). PLoS One. 2017;12(10): e0186516. https://doi.org/10.1371/journal.pone.0186516.

29. Li C, Friedman B, Conwell Y, et al. Validity of the patient health questionnaire 2 (PHQ-2) in identifying major depression in older people. J Am Geriatr Soc. 2007;55(4):596-602. https://doi.org/10.1111/j.1532-5415.2007.01103.x.

30. Fayers P, Bottomley A. Quality of life research within the EORTC - the EORTC QLQ-C30. Eur J Cancer. 2002;38:125-33. https://doi.org/10.1016/ S0959-8049(01)00448-8.

31. Andersohn F, Walker J. Characteristics and external validity of the German health risk institute (HRI) database. Pharmacoepidemiol Drug Saf. 2016;25(1): 106-9. https://doi.org/10.1002/pds.3895.

32. Raykov T. Propensity score analysis with fallible covariates. Educ Psychol Meas. 2012;72(5):715-33. https://doi.org/10.1177/0013164412440999.

\section{Publisher's Note}

Springer Nature remains neutral with regard to jurisdictional claims in published maps and institutional affiliations. 